\title{
ON THE GROBAL DIMENSION OF ORE-EXTENSIONS
}

\author{
S. M. BHATWADEKAR
}

Introduction. Let $S$ be a ring and $d$ be a derivation of $S$. The Oreextension $S(X, d)$ is the ring generated by $S$ and an indeterminate $X$ satisfying the ralation $X a-a X=d a$ for all $a$ in $S$.

It can be deduced from [3, Theorem 2] that if $S$ is a commutative noetherian ring and $d$ is a derivation of $S$, such that there exists a maximal ideal $\mathfrak{m}$ of $S$ with (i) $d(\mathfrak{m}) \subset \mathfrak{m}$ (ii) $\operatorname{gl}$. $\operatorname{dim} S=\operatorname{gl} . \operatorname{dim} S_{m}$, then l.gl. $\operatorname{dim} S(X, d)=1+$ gl. $\operatorname{dim} S$. In $\S 1$, we prove the converse of the above proposition (see theorem 1.1) if $S$ is a Dedekind ring containing field $\boldsymbol{Q}$ of rationals. This is a generalization of theorem of Rinehart [5, Propsition 2].

In $\S 2$ we compute the l.gl. $\operatorname{dim}$ of $S(X, d)$ when $S$ is a commutative noetherian ring containing $Q$ and $d$ is a derivation of $S$, such that $1 \in d(S)$ and for every $a \in S$ there exists an integer $n \geqslant 1$ such that $d^{n}(a)=0$.

My sincere thanks are due to Professor R. Sridharan for helpful suggestions.

§1. In this section we prove the following.

THeorem 1.1. Let $S$ be a Dedekind ring which contains $Q$. Let $d$ be a derivation of $S$, such that for every maximal ideal $\mathfrak{m}$ of $S, d \mathfrak{m} \not \subset \mathfrak{m}$. Then

$$
\text { l.gl. } \operatorname{dim} S(X, d)=1 \text {. }
$$

For the proof of the theorem, we need two lemmas. We start with

LEMma 1.2. Under the hypothesis of Theorem 1.1, for every maximal ideal $\mathfrak{m}$ of $S, R \mathfrak{m}$ (resp. $\mathfrak{m} R$ ) is a maximal left (resp. right) ideal of $R$, where $R$ denotes $S(X, d)$.

Proof. Let $I$ be a left ideal of $R$ such that $R \mathfrak{m} \subset I \subset R$, where $\mathfrak{m}$ 
is a maximal ideal of $S$. Suppose $I \neq R$. Then we will show that $R \mathrm{~m}=I$.

For, if not, then there exists $f \in I$ such that $f \notin R m$. Consider an element $g$ of $I$ of smallest degree and not belonging to $R \mathrm{~m}$. Without loss of generality we can take $g$ to be of the form $g=X^{k}+\sum_{0 \leqslant i \leqslant k-1} X^{i} a_{i}$, $k \geqslant 1$.

Since $d \mathfrak{m} \not \subset \mathfrak{m}$, there exists $b \in \mathfrak{m}$ such that $d b \notin \mathfrak{m}$. Consider $g^{\prime}=$ $X^{k} b-b g$. It is easy to see that $g^{\prime} \in I$ and $g^{\prime}=X^{k-1}\left(k d b-b a_{k-1}\right)+$ $\sum_{0 \leqslant i \leqslant k-2} X^{i} a_{i}^{\prime}$. This shows that $g^{\prime} \in R \mathfrak{m}$. Therefore $k d b-b a_{k-1} \in \mathfrak{m}$, i.e. $k d b \in \mathfrak{m}$. But $d b \notin \mathfrak{m}$ and $k$ is a unit in $S$. Hence we get a contradiction. Therefore $R \mathfrak{m}=I$.

This completes the proof of lemma 1.2.

LEMMA 1.3. Let $S$ and $R$ be as given in Theorem 1.1. If $J$ is a nonzero projective left ideal of $R$ and $J_{1}=J+R \phi$ for some $\phi \in R$ such that $\mathfrak{m} \phi \subset J$ for some maximal ideal $\mathfrak{m}$ of $S$, then $J_{1}$ is also a projective ideal of $R$.

Proof. $\mathrm{m} \phi \subset J$ implies that if $J \neq J_{1}$ then $J_{1} / J \simeq R / R \mathfrak{m}$. Also $J_{1} \neq J$ implies that $\operatorname{Hom}_{R}\left(J_{1}, R\right) \stackrel{\operatorname{Hom}(i, R)}{\longrightarrow} \operatorname{Hom}_{R}(J, R)$ is not a surjective map, where $i: J \rightarrow J_{1}$ inclusion map.

For, if $\operatorname{Hom}(i, R)$ is a surjective map, then $\operatorname{Hom}_{R}\left(J_{1}, F\right) \stackrel{\operatorname{Hom}(i, F)}{\longrightarrow}$ $\operatorname{Hom}_{R}(J, F)$ is surjective for every finitely generated free left module $F$ of $R$.

Let $p: F_{0} \rightarrow J$ be a surjection from a finitely generated free module $F_{0}$ on to $J$. Consider the commutative diagram

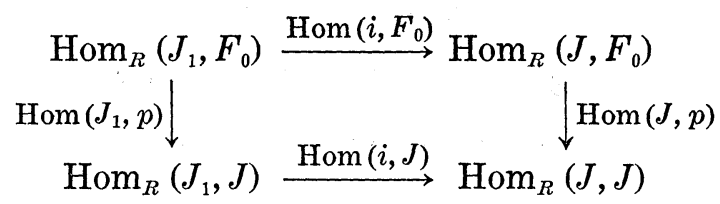

Since $J$ is a projective module, we get $\operatorname{Hom}(J, p)$ to be a surjection. Hence $\operatorname{Hom}(i, J)$ is a surjective map. This implies that $J$ is a direct summand of $J_{1}$. Since $J \neq 0$ and $J \neq J_{1}$, this gives a contradiction. Thus $\operatorname{Hom}(i, R)$ is not a surjective map.

Assume $J \neq J_{1}$. Consider the exact sequence<smiles>O[AlH][AlH][V]O</smiles>

This gives rise to an exact sequence of right $R$-modules 


$$
\begin{aligned}
\operatorname{Hom}_{R}\left(J_{1}, R\right) & \stackrel{\operatorname{Hom}\left(J_{1}, R\right)}{\longrightarrow} \operatorname{Hom}_{R}(J, R) \longrightarrow \operatorname{Ext}_{R}^{1}\left(J_{1} / J, R\right) \\
& \longrightarrow \operatorname{Ext}_{R}^{1}\left(J_{1}, R\right) \longrightarrow 0
\end{aligned}
$$

$J_{1} / J \simeq R / R \mathfrak{m}$ implies that $\operatorname{Ext}_{R}^{1}\left(J_{1} / J, R\right) \simeq \operatorname{Ext}_{S}^{1}(S / \mathfrak{m}, S) \otimes_{S} R$ as right $R$-modules. But $\operatorname{Ext}_{S}^{1}(S / \mathfrak{m}, S) \simeq S / \mathfrak{m}$. Therefore $\operatorname{Ext}_{R}^{1}\left(J_{1} / J, R\right) \simeq S / \mathfrak{m}$ $\otimes_{S} R \simeq R / \mathfrak{m} R$. By Lemma $1.2, R / \mathrm{m} R$ is a simple right $R$-module. Also, Hom $(i, R)$ is not a surjective map. Hence we get an exact sequence

$$
\operatorname{Hom}_{R}\left(J_{1}, R\right) \rightarrow \operatorname{Hom}_{R}(J, R) \rightarrow \operatorname{Ext}_{R}^{1}\left(J_{1} / J, R\right) \rightarrow 0 \text {. }
$$

This shows that $\operatorname{Ext}_{R}^{1}\left(J_{1}, R\right)=0$. By a 'direct sum' argument, we can show that $\operatorname{Ext}_{R}^{1}\left(J_{1}, F\right)=0$ for every finitely generated free left module $F$ of $R$.

Let $M$ be a finitely generated left module of $R$. Let $0 \rightarrow C \rightarrow F \rightarrow$ $M \rightarrow 0$ be an exact sequence of left $R$-modules where $F$ is free module of finite rank.

Then we get an exact sequence

$$
0=\operatorname{Ext}_{R}^{1}\left(J_{1}, F\right) \rightarrow \operatorname{Ext}_{R}^{1}\left(J_{1}, M\right) \rightarrow \operatorname{Ext}_{R}^{2}\left(J_{1}, C\right) .
$$

But we know that $1 . g l . \operatorname{dim} R \leqslant 2$. Also, since $R$ is not semisimple, from [1, Theorem 1] it follows that

$$
\text { l.gl. } \operatorname{dim} R=1+\sup _{I} \text { hd. I . }
$$

where $I$ ranges over all left ideals of $R$.

Therefore hd. $I \leqslant 1$ for every left ideal $I$ of $R$. This gives $\operatorname{Ext}_{R}^{2}\left(J_{1}, C\right)$ $=0$. Therefore $\operatorname{Ext}_{R}^{1}\left(J_{1}, M\right)=0$. Thus for every finitely generated $R$ module $M$ we get $\operatorname{Ext}_{R}^{1}\left(J_{1}, M\right)=0$. This proves that $J_{1}$ is a projective left ideal of $R$.

If $J=J_{1}$ then there is nothing to prove.

Thus the proof of Lemma 1.3 is complete.

Proof of Theorem 1.1. Let $R$ denote $S(X, d)$. From [1, Theorem 1] it follows that it is enough to prove that every left ideal of $R$ is projective.

Let $I$ be a left ideal of $R$. For any integer $k \geqslant 0$ let

$$
I_{k}=\left\{a \mid \begin{array}{l}
a \in S, \text { such that } a \text { is leading coefficient } \\
\text { of some element of } I \text { of degree } k
\end{array}\right\} .
$$

Then it is easy to see that we get an increasing sequence $I_{0} \subset I_{1} \subset I_{2} \ldots$ 
of ideals of $S$. Let $m$ be the least integer such that $I_{m}=I_{n}$ for $n \geqslant m$. Let $k_{0}$ be the least integer such that $I_{k_{0}} \neq 0$. Let $\left(b_{k}^{1}, \cdots, b_{k}^{n_{k}}\right)$ be a set of generators of $I_{k}$ for $k_{0} \leqslant k \leqslant m$. By definition of $I_{k}$, there exist elements $\left(f_{k}^{1}, \cdots, f_{k}^{n_{k}}\right)$ of $I$ such that $f_{k}^{i}$ is of degree $k$ and with leading coefficient $b_{k}^{i}$ for every $i, 1 \leqslant i \leqslant n_{k}$.

Let $J_{k}=\sum R f_{l}^{i}, 1 \leqslant i \leqslant n_{l}, k_{0} \leqslant l \leqslant k$. Then we get an increasing sequence $0 \neq J_{k_{0}} \subset \cdots \subset J_{m}$ of left ideals of $R$ such that $J_{m}=I$. It is easy to prove that $J_{0} \simeq R \otimes_{S} I_{k_{0}}$ as left ideals of $R$.

Let $r=m-k_{0}$. We will prove the result by induction on $r$.

If $r=0$, then $I=J_{m}=J_{k_{0}} \simeq R \otimes_{S} I_{k_{0}}$. Since $S$ is a Dedekind ring, $I_{k_{0}}$ is a projective ideal of $S$. This shows that $I$ is a projective left ideal of $R$.

Assume the result for $r-1 \geqslant 0$. Then by induction hypothesis $J_{m-1}$ is a projective left ideal of $R$. Since $I_{m-1} \neq 0$, there exists an increasing sequence

$I_{m-1}=\mathscr{B}_{0} \subset \mathscr{B}_{1} \subset \mathscr{B}_{2} \ldots \mathscr{B}_{p}=S$ of ideals of $S$ such that $\mathscr{B}_{i} / \mathscr{B}_{i-1} \simeq S / \mathfrak{m}_{i}$ for some maximal ideal $\mathfrak{m}_{i}$ of $S, 1 \leqslant i \leqslant p$.

Therefore $\mathscr{B}_{i}=\mathscr{B}_{i-1}+S \theta_{i}$ for some $\theta_{i} \in S$. We can take $\theta_{p}=1$. Then there exists a maximal ideal $\mathfrak{m}_{i}$ such that $\mathfrak{m}_{i} \theta_{i} \subset \mathscr{B}_{i-1}$. Let $\mathscr{A}_{i}^{j}=J_{m-1}+$ $R\left(f_{m}^{1}, \cdots, f_{m}^{i-1}\right)+R \theta_{1} f_{m}^{i}+R \theta_{2} f_{m}^{i}+\cdots+R \theta_{j} f_{m}^{i}, 1 \leqslant i \leqslant n_{m}, 1 \leqslant j \leqslant p . \quad$ Then $\mathscr{A}_{i}^{j} \subset \mathscr{A}_{l}^{k}$ if either $i \leqslant l$, or $i=l$ and $j \leqslant k$. Also $\mathscr{A}_{n_{m}}^{p}=J_{m}$. From the definition of $\mathscr{A}_{i}^{j}$ it follows that either $\mathscr{A}_{i}^{j}=\mathscr{A}_{i}^{j+1}$ or $\mathscr{A}_{i}^{j+1} / \mathscr{A}_{i}^{j} \simeq R / R \mathrm{~m}$ for some maximal ideal $m$ of $R$. Also, either $\mathscr{A}_{1}^{1}=J_{m-1}$ or $\mathscr{A}_{1}^{1} / J_{m-1} \simeq$ $R / R \mathfrak{m}$. Since $J_{m-1}$ is $R$-projective by our assumption, by using Lemma 1.3 step by step, we get $J_{m}(=I)$ is a projective left ideal of $R$.

This proves theorem 1.1.

Remark. Theorem 1.1 shows that if $S$ is a Dedekind ring containing $\boldsymbol{Q}$ and $d$ is a derivation of $S$ then

$$
\text { l.gl. } \operatorname{dim} . S(X, d)=2=1+\text { gl. } \operatorname{dim} . S \text { iff }
$$

there exists a maximal ideal $\mathfrak{m}$ of $S$ such that $d \mathfrak{m} \subset \mathfrak{m}$.

§2. In this section we prove the following theorem.

THEOREM 2.1. Let $S$ be a commutative noetherian ring of global dimension $n<\infty$, such that $Q \subset S$. Let $d$ be a derivation of $S$ such that $1 \in d(S)$ and for every $a \in S$ there exists an integer $k \geqslant 1$ such that $d^{k}(a)=0$ then 
l.gl. $\operatorname{dim} S(X, d)=n$.

First we state a lemma. [4, p. 78].

LEMMA 2.2. Under the hypothesis of Theorem 2.1, if $d(b)=1$ for $b \in S$, then the mapping

$$
\begin{gathered}
\chi: S \rightarrow(S / S b)[Y] \\
\chi(a)=\bar{a}+\overline{d a} Y+\overline{d^{2} a} \quad Y^{2} / 2 !+\overline{d^{3} a} \quad Y^{3} / 3 !+\cdots
\end{gathered}
$$

is an isomorphism of rings, where $\overline{d^{i} a}$ denotes the image of $d^{i} a$ in $S / S b$ under the canonical mapping $\eta: S \rightarrow S / S b$.

Moreover, if $D$ is the $S / S b$-derivation of $S / S b[Y]$ given by $D Y=1$, then $\chi$ is an isomorphism of differential rings.

This shows that it is sufficient to prove the theorem if $S=A[Y]$ where $A$ is a commutative noetherian ring of finite global dimension which contains $Q$ and $d$ is the $A$-derivation of $S$ given by $d Y=1$. Also it is easy to see that it is enough to prove the result in case $A$ is a local ring.

So we prove the following theorem.

THEOREM 2.3. Let $A$ be a commutative noetherian local ring of global dimension $n<\infty$ such that $\boldsymbol{Q} \subset A$. Let $S=A[Y]$ and $d$ be the A-derivation of $S$ given by $d Y=1$. Then

$$
\text { l.gl. } \operatorname{dim} S(X, d)=n+1 \text {. }
$$

Before proceeding further we will give some definitions and results which can be found in $[7, \S 15]$.

Let $B$ be a ring, not necessarily commutative. Let $T$ be a multiplicatively closed subset of $B$ such that $1 \in T$.

Definition. $T$ is called right (resp. left) permutable if given $a \in B$ and $t \in T$, there exist $b \in A$ and $s \in T$ such that $t b=a s$ (resp. $b t=s a$ ).

DEFINITION. $T$ is called right (resp. left) reversible if $t a=0$ (resp. $a t=0$ ) with $t \in T, a \in B$ implies $a s=0$ (resp. $s a=0$ ) for some $s \in T$.

DEFINITION. A right (resp. left) ring of fractions of $B$ with respect to $T$ is a ring $B\left[T^{-1}\right]$ (resp. $\left[T^{-1}\right] B$ ) and a ring homomorphism $\phi: B \rightarrow B\left[T^{-1}\right]$ (resp. $\psi: B \rightarrow\left[T^{-1}\right] B$ ) satisfying

i) $\phi(s)$ (resp. $\psi(s)$ ) is invertible for every $s \in T$. 
ii) every element in $B\left[T^{-1}\right]$ (resp. $\left[T^{-1}\right] B$ ) has the form

$$
\left.\phi(a) \phi(s)^{-1} \text { (resp. } \psi(s)^{-1} \psi(a)\right) \quad \text { with } s \in T \text {. }
$$

iii) $\phi(a)=0$ (resp. $\psi(a)=0$ ) iff $a s=0$ (resp. $s a=0$ ) for some $s \in T$. Some results concerning $B\left[T^{-1}\right]$.

(a) If $B\left[T^{-1}\right]$ exists, it is unique up to isomorphism.

(b) $B\left[T^{-1}\right]$ exists iff $T$ is right permutable and right reversible set.

(c) $B\left[T^{-1}\right]$ is $B$-flat as a left $B$-module.

(d) If both $B\left[T^{-1}\right]$ and $\left[T^{-1}\right] B$ exist, they are isomorphic.

We have similar results for $\left[T^{-1}\right] B$.

Definition. $A$ ring $B$ is said to be left coherent if every finitely generated left ideal of $B$ is finitely presented.

Let w.gl. $\operatorname{dim} B$ denote the weak global dimension of $B$. If $B$ is left noetherian then 1.gl. $\operatorname{dim} B=$ w.gl. $\operatorname{dim} B . \quad$ [2, Chapt. VI].

The proof of Theorem 2.3 depends upon the following proposition.

Proposition 2.4. Let $\left(T_{i}\right)_{i \in I}$ be a finite family of multiplicatively closed subsets of a ring $R$ such that

(i) Each $T_{i}$ is right permutable and right reversible.

(ii) For every family $\left(t_{i}\right)_{i \in I}$ of elements of $R$ with $t_{i} \in T_{i}$ we have $\sum_{i \in I} t_{i} R=R$.

(iii) Every $R_{i}$ is $R$-flat as a left $R$-module and as a right $R$-module, where $R_{i}=R\left[T_{i}^{-1}\right]$

(iv) w.gl. $\operatorname{dim} R<\infty$

(v) $R$ is left coherent.

Then w.gl. $\operatorname{dim} R \leqslant \sup _{i \in I}$ w.gl. $\operatorname{dim} R_{i}$.

For a proof, see [6, Proposition 1].

Proof of Theorem 2.3. Let $R$ denote the ring $S(X, d)$. Then under the hypothesis of Theorem 2.1, $R$ is nothing but the $A$-algebra $A\{X, Y\}$ in two variables $X$ and $Y$ and with the relation $X Y-Y X=1$.

Let $\mathfrak{m}$ be the maximal ideal of $A$. Let $T_{1}=A[X]-\mathfrak{m}[X]$ and $T_{2}=$ $A[Y]-\mathfrak{m}[Y]$ be two multiplicatively closed subsets of $R$.

Since $S(X, d)$ is without proper divisors of zero $T_{1}$ and $T_{2}$ are right as well as left reversible.

To prove that $T_{1}$ is right permutable it is enough to show that given $f$ in $T_{1}$ and $Y^{n}$ there exist $g$ in $T_{1}$ and $h$ in $S(X, d)$ such that $Y^{n} g=f h$. Taking $g=f^{n+1}$ we see that $Y^{n} f^{n+1}=\sum_{0 \leqslant i \leqslant n}{ }^{n} C_{i} d^{i}\left(f^{n+1}\right) Y^{n-i}$. But 
$d^{i}\left(f^{n+1}\right)=f h_{i}$ for some $h_{i}$ in $A[X]$. Therefore $Y^{n} f^{n+1}=f h$ where $h=$ $\sum_{0 \leqslant i \leqslant n}{ }^{n} C_{i} h_{i} Y^{n-i}$.

Similarly we prove that $T_{1}$ is left permutable and $T_{2}$ is right and left permutable. This shows that $R\left[T_{i}^{-1}\right]$ is $R$-flat as a right $R$-module as well as left $R$-module for every $i=1,2$.

Since $R$ is left noetherian and l.gl. $\operatorname{dim} R \leqslant n+2$ we see that all the conditions of the previous proposition except the second condition are satisfied.

Assume for the time being that the second condition is also satisfied. Then

$$
\text { w.gl. } \operatorname{dim} R \leqslant \max _{i} \text { w.gl. } \operatorname{dim} R_{i}
$$

when $R_{i}=R\left[T_{i}^{-1}\right]$.

Let $d$ be the $A$-derivation of $A[Y]$ given by $d Y=1$. If $S^{\prime}$ is the localization of $A[Y]$ with respect to the prime ideal $m[Y]$ and $d^{\prime}$ is the derivation of $S^{\prime}$ induced by $d$ then $R\left[T_{2}^{-1}\right]$ is nothing but the Ore-extension of $S^{\prime}$ with respect to $d^{\prime}$. Hence w.gl. $\operatorname{dim} R\left[T_{2}^{-1}\right]=1 . g l . \operatorname{dim} R\left[T_{2}^{-1}\right] \leqslant$ $1+$ gl. $\operatorname{dim} S^{\prime}$. But gl. $\operatorname{dim} S^{\prime}=n$. Therefore w.gl. $\operatorname{dim} R\left[T_{2}^{-1}\right] \leqslant n+1$.

Similarly we can show that w.gl. $\operatorname{dim} R\left[T_{1}^{-1}\right] \leqslant n+1$.

Hence w. gl. $\operatorname{dim} R \leqslant n+1$. But we already know that $n+1 \leqslant$ 1.gl. $\operatorname{dim} R=$ w.gl. $\operatorname{dim} R$.

Hence the equality.

The lemma given below shows that $T_{1}$ and $T_{2}$ satisfy the second condition of the proposition.

LEMMA 2.5. Under the hypothesis of Theorem 2.3, if $f \in T_{1}$ and $g \in T_{2}$ then $f R+g R=R$.

Proof. We will prove the result by using induction on the global dimension of $A$.

If $\operatorname{gl} \operatorname{dim} A=0$, then $A$ is a field of char $=0 . \quad$ The result in this case is proved in $[6, \mathrm{p} .25-26]$.

Assume the result for $n-1$. Let gl. $\operatorname{dim} A=n$. If $\mathscr{B}=f R+g R$ then by our induction hypothesis there exists an integer $r \geqslant 1$ such that $\mathfrak{m}^{r} \subset \mathscr{B} \cap A$, where $\mathfrak{m}$ is the maximal ideal of $A$. (Since for every prime ideal $\mathfrak{p}$ of $A$ other than $\mathfrak{m}, \mathscr{B}_{\mathfrak{p}}=R_{\mathfrak{p}}$.) We will prove that $A \subset \mathscr{B} \cap A$ by proving that $\mathfrak{m}^{r-1} \subset \mathscr{B} \cap A$.

Let $a \in \mathfrak{m}^{r-1}$. We can write $f=f_{0}+f_{1}$ and $g=g_{0}+g_{1}$ where all 
the coefficients of $f_{1}$ and $g_{1}$ are in $m$ and all nonzero coefficients of $f_{0}$ and $g_{0}$ are units in $A$. Because of the choice of $f$ and $g$ we get $f_{0} \neq 0$, $g_{0} \neq 0$.

Since $\mathfrak{m}^{r} \subset \mathscr{B} \cap A, f_{1} a \in \mathscr{B}$ and $g_{1} a \in \mathscr{B}$. This shows that $f_{0} \cdot a \in \mathscr{B}$ and $g_{0} \cdot a \in \mathscr{B}$. We will prove $a \in \mathscr{B}$ by showing that $f_{0} R+g_{0} R=R$.

Let $\hat{A}$ be the completion of $A$ with respect to the m-adic topology. $\hat{A}$ contains a subfield $k$ isomorphic to $A / \mathrm{m}$.

We can regard $f_{0}$ and $g_{0}$ as elements of $k[X]$ and $k[Y]$ respectively. Since char $k \neq 0$, there exist $h_{1}$ and $h_{2}$ in $k\{X, Y\}$ such that $f_{0} h_{1}+g_{0} h_{2}=1$. This shows that $f_{0} \hat{R}+g_{0} \hat{R}=\hat{R}$ where $\hat{R}=\hat{A} \otimes_{A} R$.

Let $\mathscr{A}=f_{0} R+g_{0} R$. Since $\hat{A}$ is faithfully flat over $A$, and since we have $R / \mathscr{A} \otimes_{A} \hat{A}=\widehat{R / \mathscr{A}}=0$, we get $R / \mathscr{A}=0$, i.e. $f_{0} R+g_{0} R=R$. Therefore $a \in \mathscr{B}=f R+g R$. This shows that $A \subset \mathscr{B} \cap A$ i.e. $\mathscr{B}=R$. This completes the proof of Lemma 2.5.

The proof of Theorem 2.4 is complete.

CoRollary 2.6. Let $A_{n}(S)=S\left\{X_{1}, \cdots, X_{n}, \partial / \partial X_{1}, \cdots, \partial / \partial X_{n}\right\}$ be the Weyl algebra of index $n$ with coefficients in $S$, where $S$ is a commutative noetherian ring which contains $\boldsymbol{Q}$. Then

$$
\text { gl. } \operatorname{dim} A_{n}(S)=n+\text { gl. } \operatorname{dim} S .
$$

Proof of Corollary 2.6. We will prove the result by induction on $n$. Theorem 2.3 proves the result when $n=1$. Assume the result for $n-1$.

Let $A_{n}(S)=S\left\{X_{1}, \cdots, X_{n}, \partial / \partial X_{1}, \cdots, \partial / \partial X_{n}\right\}$. We can assume without loss of generality that $S$ is a regular local ring with maximal ideal $\mathrm{m}$. Let $T_{1}=S\left[X_{n}\right]-\mathfrak{m}\left[X_{n}\right]$ and $T_{2}=S\left[\partial / \partial X_{n}\right]-\mathfrak{m}\left[\partial / \partial X_{n}\right]$ be the multiplicatively closed sets satisfying the conditions of the Proposition 2.4.

If $B=S\left\{X_{1}, \cdots, X_{n}, \partial / \partial X_{1}, \cdots, \partial / \partial X_{n-1}\right\}$, then $T_{1}$ consists of central elements of $B$. Therefore the $S$-derivation of $B$ given by $\partial / \partial X_{n}$ can be extended to a derivation $d^{\prime}$ of $B\left[T_{1}^{-1}\right]$. Since $A_{n}(S)$ is the Ore-extension of $B$ with respect to derivation $\partial / \partial X_{n}, A_{n}(S)\left[T_{1}^{-1}\right]$ is the Ore-extension of $B\left[T_{1}^{-1}\right]$ with respect to the derivation $d^{\prime}$. Therefore l.gl. $\operatorname{dim} A_{n}(S)\left[T^{-1}\right]$ $\leqslant 1+$ l.gl. $\operatorname{dim} B\left[T_{1}^{-1}\right]$.

But $B\left[T_{1}^{-1}\right] \simeq S^{\prime}\left\{X_{1}, \cdots, X_{n-1}, \partial / \partial X_{1}, \cdots, \partial / \partial X_{n-1}\right\}$ where $S^{\prime}$ is the localization of $S\left[X_{n}\right]$ with respect to $T_{1}$. Therefore by induction hypothesis 1.gl. $\operatorname{dim} B\left[T_{1}^{-1}\right]=n-1+\operatorname{gl} . \operatorname{dim} S^{\prime}=n-1+\operatorname{gl} . \operatorname{dim} S . \quad$ This shows that l.gl. $\operatorname{dim} A_{n}(S)\left[T_{1}^{-1}\right] \leqslant n+\operatorname{gl} \cdot \operatorname{dim} S$. Similarly we prove that 
l.gl. $\operatorname{dim} A_{n}(S)\left[T_{2}^{-1}\right] \leqslant n+$ gl. $\operatorname{dim} S$. Therefore by Proposition 2.4 we get that 1.gl. $\operatorname{dim} A_{n}(S) \leqslant n+\operatorname{gl} \cdot \operatorname{dim} S$. But we already know that l.gl. $\operatorname{dim} A_{n}(S) \geqslant n+$ l.gl. $\operatorname{dim} S$. Hence the equality.

Remark. Theorem 2.3 is a generalization of a Theorem of Rinehart [5, Proposition 2].

Remark. Corollary 2.6 is a generalization of a Theorem of Roos [6, Theorem 1].

\section{REFERENCES}

[1] M. Auslander, On the Dimension of Modules and algebras (III). Global dimension, Nagoya Mathematical Journal, 9, 67-77 (1955).

[2] Cartan, H. and S. Eilenberg, Homological Algebra, Princeton University Press, 1956.

[ 3 ] N. S. Gopalakrishnan and R. Sridharan, Homological Dimension of Ore-extensions, Pacific J. Math., 19, 67-75 (1966).

[4] Y. Mouaze and P. Gabriel, Idéaux Premiers de l'Algebre Enveloppante d'une Algèbre de lie Nilpotente, J. of Algebra, 6, 77-99 (1967).

[ 5 ] Rinehart, G. S., Note on the Global Dimension of certain rings, Proc. Amer. Math. Soc., 13, 341-346 (1962).

[ 6 ] M. Jan-Erik Roos, Dètermination de la dimension homologique globale des algèbres de Weyl, Comptes Rendus, Séries A, 274, 23-26 (1972).

[ 7 ] Bo Stenström, Rings and Modules of Quotients, Springer, Berlin, 1971.

School of Mathematics

Tata Institue of Fundamental Research 